\title{
SAATY'S CONSISTENCY ANALYSIS: AN APPLICATION TO PROBLEMS IN STATIC AND DYNAMIC INPUT-OUTPUT MODELS
}

\author{
ALBERT E. STEENGE \\ Twente University of Technology, Department of Public Administration, P.O. Box 217, \\ 7500 AE Enschede, The Netherlands
}

(Received 8 October 1985; in revised form 26 December 1985)

\begin{abstract}
Saaty's priority analysis has given us a set of rules to assign weights (priorities) to a multiple of objects in a consistent way. The method has been shown to have great potential for economic application, especially in applied input-output work. In this paper we show that much stronger links exist between priority theory and input-output analysis than observed in the literature thus far. As we shall show, application of Saaty's instrumentarium to the Leontief framework opens the way for a new type of consistency analysis. In static models the dual output and price systems are shown to be consistent, in the sense that both can be described in terms of the same pairwise comparison matrix. In dynamic analysis, these dual systems are shown to be generally inconsistent, the only exception being the case of von Neumann growth.
\end{abstract}

\section{INTRODUCTION}

In a series of publications, T. L. Saaty and others [1, $2,3]$ have shown that hierarchical measurement can be applied to a number of problems in input-output analysis. In fact, it has convincingly been shown that this approach can furnish quick and remarkably reliable estimates of major input coefficients. The potentialities of consistency analysis become quite clear by a comparison of Saaty and Vargas' work on the Sudan with a model developed along traditional lines by Klein and others $[1,2]$. This fact alone already signals an important breakthrough, because data gathering and manipulation often require a disproportional amount of time in applied work, causing severe publication lags (see, e.g. Taylor [4]). Therefore, the development of techniques that may speed up this process is of utmost importance.

However, Saaty's approach may be of additional significance for quite different reasons. Input-output analysis presents an equilibrium view of the economy. That is, the Walrasian invisible hand (or any other mechanism that we may wish to distinguish) has done its job in equalizing supply and demand. Prices in Leontief analysis thus reflect the relative importance that society attaches to each of its goods being produced. The Leontief framework, however, is a rather peculiar framework in the sense that price and real output relations exhibit a strict duality. This means, among other things, that these systems can be solved and analyzed independently from each other.

For static models this causes no problems. For dynamic systems the situation is quite different, because they are invariably plagued by the phenomenon of "dual-instability." That is, if the real output system converges to the proportions of its von Neumann ray, the price system may become meaningless because it generates negative prices or otherwise economically meaningless outcomes. (A vice-versa result also holds here.) A number of alternative specifications have been offered, none of which throws much light on the origins of the problem (see also Section 5). Because, in our view, it basically is a problem of consistency between output and price relations, Saaty's system might be of help here. For example, it is evident that if all commodities (including labour) have been consistently priced (in a yet to be specified sense), then also pairwise comparisons must reveal the same consistency.

A problem now arises. As is well-known, a major tool in hierarchical analysis is Saaty's priority matrix, a reciprocal matrix having unit rank if all priorities are assigned consistently. Because of its fundamental character, we might expect the reciprocal matrix also to turn up in certain economic equilibrium models. However, the real situation is quite different. In fact, the "typical" Saaty tools, viz. eigenvalues and eigenvectors, hardly play any role at all in the input-output mechanism. One of the few places encountered is a qualitative statement that productivity of open systems is assured only if the Frobenius eigenvalue of the matrix of input coefficients is smaller than unity. In dynamic analysis, their role essentially is limited to an analysis of von Neumann type paths of balanced growth as certain reference paths. It is clear that additional insight in this matter also might help explain the relevance of Saaty's system for input-output economics, and enhance its acceptance among the profession.

In this paper we shall show that the Leontief equations, in fact, can be rewritten in a form containing Saaty's pairwise comparison reciprocal matrix. This, however, requires a certain reformulation of the model, where primitivity of the input matrix is seen to play a role. As will be shown, this reformulation, in itself, generates new insights. For example, we now are able to show that commodity bundles of particular composition play a major role in the price and output relations. Also new insights in certain dynamic problems are obtained. This then may be the deeper point of this paper: It may pay, in analyzing equilibrium models, to look for formalizations that incorporate the basics of priority theory.

\section{INDIRECT INPUTS IN OPEN SYSTEMS}

As is well-known, powers of the matrix of input coefficients $T$ play an important role in the open inputoutput model

$$
\begin{aligned}
& \mathbf{x}=\mathbf{T x}+\mathbf{f} \\
& L=\mathbf{l x}
\end{aligned}
$$

with $\mathbf{T}$ and $\mathbf{I}$, resp., the indecomposable matrix of input 
coefficients and the vector of direct labour input coefficients, $f$ and $x$ the vectors of final demand and total outputs, and $L$ the required labour force. We shall assume labour to be indispensable in production $(\mathbf{l}>0)$, and $\mathbf{f}$ to be semipositive ( $\mathbf{f} \geq 0$ ). Total output is obtained by solving for $\mathbf{x}$

$$
\mathbf{x}=(\mathbf{I}-\mathbf{T})^{-1} \mathbf{f}
$$

Equilibrium prices are obtained from the dual system

$$
\mathbf{p}=\mathbf{p} \mathbf{T}+w \mathbf{l},
$$

or

$$
\mathbf{p}=w \mathbf{l}(\mathbf{I}-\mathbf{T})^{-1} \equiv w \mathbf{l}^{*}
$$

where $w$ is the wage rate. Here $I^{*}$ is traditionally known as the vector of the embodied labour times. (Therefore, these prices are often called "labour theory of value" prices. See, e.g. Dosso [5], Takayama [6], and Miller and Blair [7].) We see that in the above scheme, all inputs, labour included, are imputed to the production of the final demand vector $\mathbf{f}$. Prices reflect the "total" input requirements (of commodities and labor) needed to produce a unit of exogenously determined final demand $f$.

The Leontief inverse $(\mathbf{I}-\mathbf{T})^{-1}$ can be written as an infinite sum:

$$
(\mathbf{I}-\mathbf{T})^{-1}=\mathbf{I}+\mathbf{T}+\mathbf{T}^{2}+\cdots
$$

where $\lim _{t \rightarrow \infty} \mathbf{T}^{t}=0$. Here Dosso [5, p. 234] comments: "In a general Leontief model, everything is needed for everything. There are no early and late stages, no previous stages, no higher or lower stages. Coal is needed to produce fertilizer; fertilizer is needed to produce coal. Neither is prior to the other." The powers of $\mathbf{T}$ thus simply reflect the circulatory of the system, and make us realize that production (per year, say) involves not only the fertilizer to produce coal, but also the fertilizer to produce the steel to produce coal, etc.

Here, $\mathbf{T}$ is nonnegative and indecomposable, which implies that all goods are needed, directly or indirectly, in the production of each good. However, indecomposable matrices come in two types; they can be primitive (noncyclic) and imprimitive (cyclic). It is not difficult to see why the property of primitivity plays no role in the above traditional set-up. Prices being proportional to $\mathrm{I}^{*} \equiv \mathbf{l}(\mathbf{I}-\mathbf{T})^{-1}$, are always positive. Thus, for example, no problems of interpretation will arise due to the presence of, say, negative elements. Also, $\lim _{t \rightarrow \infty} \mathbf{T}^{t}=0$, irrespective of (im)primitivity of $\mathbf{T}$. So, at first sight, there seems no reason to include a discussion of primitivity of the input matrix.' However, the system can be analyzed in a quite different way in which (im)primitivity does play a role. Exploring this alternative will immediately reveal a direct link with Saaty's priority theory. First, however, we shall introduce the principles of the method employing closed systems in the next section. Hereafter, we shall come back to opcn systems.

\section{CLOSED SYSTEMS}

A discussion of indirect input coefticients, vital in interpreting the open system, is virtually absent in dis- cussions of input-output systems of the closed form

$$
\mathbf{z}=\mathbf{M} \mathbf{z}
$$

with $\mathbf{M}$ the matrix of direct input coefficients (having Frobenius eigenvalue equal to unity), and $\mathbf{z}$ the vector of sectoral outputs. The accompanying dual price equation is given as

$$
\mathbf{y}=\mathbf{y M}
$$

which simply defines relative prices $\mathbf{y}$ as elements of the lefthand Frobenius eigenvector of $\mathbf{M}$. As is wellknown, the price of any good can be chosen as the numeraire to obtain absolute prices. As far as we know, there is no literature on efforts to look for additional insight by introducing notions of indirect input coefficients in this closed model. Yet such coefficients can be quite easily obtained.

In Section 2 we mentioned that we may look upon total production as the summation of the desired final demand $f$, the direct inputs to produce $f$, the direct inputs to produce the direct inputs to produce $f$, etc. Here the situation is clearly different. However, a moment's reflection will show us that in closed systems a multiplicative procedure is available to obtain insight in the properties of indirect inputs. To see this, let us consider the system's "second round" or "second order" inputs (cf. matrix $\mathbf{T}^{2}$ in the open model of Section 2). If also in (3.1) fertilizer and coal are distinguished as separate categories, this would mean that we like to consider what we can say about the fertilizer to produce the fertilizer to produce the coal plus the fertilizer to produce the coal to produce the coal plus the fertilizer to produce the steel to produce the coal, etc. We immediately see that if fertilizer is the $i$ th good and coal the $j$ th, we are in fact discussing the $(i, j)$ th element of a new matrix of input coefficients, $\mathbf{M}^{2}$. We now observe that total production $\mathbf{z}$ can very well be described in terms of these indirect inputs: $\mathbf{M}^{2} \mathbf{z}=\mathbf{M}(\mathbf{M z})=\mathbf{M z}$ $=\mathbf{z}$. (Similar arguments are available for the price equation: $\left.\mathbf{y} \mathbf{M}^{2}=\mathbf{y M}(\mathbf{M})=\mathbf{y} \mathbf{M}=\mathbf{y}\right)$. Let us denote the $(i, j)$ th element of $\mathbf{M}^{2}$ by the symbol $m_{i j}^{(2)}$, and let us, to distinguish them from the traditional "first order" coefficients $m_{i j}$, call them "second order" input coefficients.

The "second order" coefficients are the elements of matrix $\mathbf{M}^{2}$. However, we can continue and investigate even higher order coefficients. So, proceeding, the elements $m_{i j}^{(3)}$ of $\mathbf{M}^{3}$ then represent "third order" inputs, etc. In this way, a class of such input matrices can be defined, each member of which incorporates more indirect inputs than the previous one. How far can one go on? Here we need the following fundamental theorem, going back to Markov:

Theorem 1 . Let $\mathbf{M}$ be a nonnegative, indecomposable and primitive $(n, n)$-matrix with Frobenius eigenvalue equal to unity. Then $\mathbf{M}^{\infty} \equiv \lim _{t \rightarrow \infty} \mathbf{M}^{t}=z \cdot \mathbf{y}$, where $z$ and $y$ are normalized right- and left-hand Frobenius eigenvectors of $\mathbf{M}$, such that $\mathbf{y} \cdot z=1$.

Proof. Well-known.

Hence, the limit of the sequence $\mathbf{M}, \mathbf{M}^{2}, \mathbf{M}^{3}, \ldots$, where $\mathbf{M}$ is primitive, gives us "infinite order" input coefficients for the closed system $\{(3.1),(3.2)\}$. Here "infinite order" means that in these input coefficients, all in- 
direct inputs are accounted for. The output vector $\mathbf{z}$ thus can be written correspondingly in terms of these coefficients as

$$
\mathbf{z}=\mathbf{M}^{\infty} \mathbf{z} .
$$

Corresponding to (3.2), we then have

$$
\mathbf{y}=\mathbf{y} \mathbf{M}^{\infty}
$$

Let us take the infinite power $\mathbf{M}^{\alpha}$ to base a definition of "embodiedness". We obtain:

Definition 1. Let $\mathbf{M}$ be an indecomposable primitive matrix of input coefficients with Frobenius eigenvalue equal to unity. Then the amount of good $i$ embodied in the production of one unit of good $j$ is given by $m_{i j}^{(\infty)}$, the $(i, j)$ th element of matrix $\mathbf{M}^{r i t}=\lim _{t \rightarrow \infty} \mathbf{M}^{t}$. Written out, we have

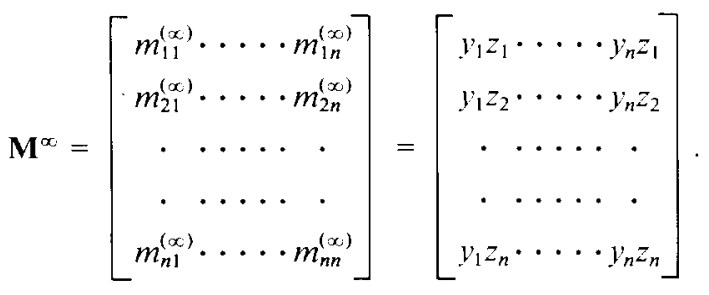

We see that each column of the rank one matrix $\mathbf{M}^{\infty}$ is proportional to $z$, the total output vector. In fact, for the $j$ th column $m_{j}^{(\infty)}$ we may write $m_{j}^{(\infty)}=y_{j} \cdot z$, with $y_{j}$ the $j$ th element of $\mathbf{y}$. So, each price $y_{j}$ reflects the (standardized) fraction of $z$ (i.e. $z$ ), ultimately used up in the production of one unit of good $j$. But this is equivalent to saying that the bundle $z$ can be treated as a numeraire as well. We shall call such a numerairebundle a composite numeraire. (Wc obscrve again that labour has no special role in this model. The final demand sector is treated as if it were an ordinary industry: Human consumption is viewed as inputs in a process producing "labour." Objections to this mechanistic view of the elements of the final consumption vector can be found in Dosso [5, pp. 245-248].)

As a consequence of the above, the output system (3.3), written out, reads:

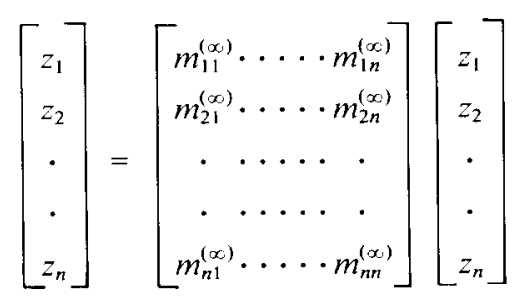

$\mathbf{M}^{\infty}$ is a matrix of rank one, its elements can be viewed as indicators of the "weights" of each type of commodity in each unit production. However, $\mathbf{M}^{\infty}$ is not (yet) a matrix of pairwise comparisons of weights as used in priority theory. Nevertheless, the system can be straightforwardly rewritten in terms of a Saaty priority matrix, which, clearly, also has rank one. Noting that $\left(3.3^{\prime}\right)$ is in terms of the embodied amount (cf. Def. 1) of each good per unit of production of each of the $n$ sectors, rewriting the system in such a way that the embodied amount of the "own" good is standardized at unity, we immediately have: $\left[\begin{array}{c}z_{1} \\ z_{2} \\ \cdots \\ \cdots \\ z_{n}\end{array}\right]$

$$
=\left[\begin{array}{ccc}
1 & \cdots \cdots & m_{1 n}^{(\infty)} / m_{n n}^{(\infty)} \\
m_{21}^{(\infty)} / m_{11}^{(\infty)} & \cdots & m_{2 n}^{(\infty)} / m_{n n}^{(\infty)} \\
\cdots & \cdots & \cdots \\
\cdots & \cdots & \cdots \\
m_{n 1}^{(\infty)} / m_{11}^{(\infty)} \cdots & \cdots & 1
\end{array}\right]\left[\begin{array}{c}
\left(m_{11}^{(\infty)}\right) z_{1} \\
\left(m_{22}^{(\infty)}\right) z_{2} \\
\cdots \\
\cdots \\
\left(m_{n n}^{(\infty)}\right) z_{n}
\end{array}\right]
$$

or

$\left[\begin{array}{c}z_{1} \\ z_{2} \\ \cdots \\ \cdots \\ z_{n}\end{array}\right]$

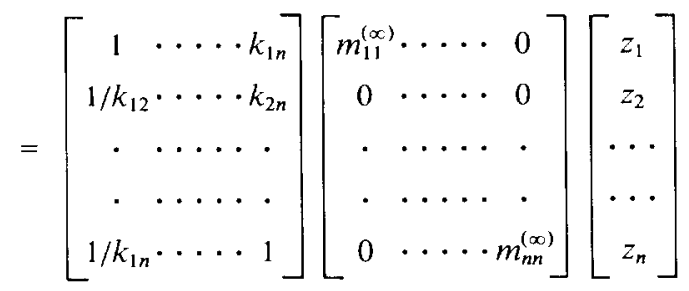

with $k_{i j} \equiv m_{i j}^{(\infty)} / m_{j j}^{(\infty)}$, and $i, j=1, \ldots, n$. Or, equivalently,

$$
\mathbf{z}=[\mathbf{K} \cdot \hat{\mathbf{D}}] \mathbf{z},
$$

with $\mathbf{K}$ the rank one priority matrix and $\hat{\mathbf{D}}$ $\equiv \operatorname{Diag}\left(m_{i i}^{(\infty)}\right), i=1, \ldots, n$. In the next section we shall show that a similar framework can be developed for open systems as well. Also here, Saaty consistency analysis points to the existence of composite numeraires.

\section{OPEN SYSTEMS}

In this section we shall discuss the standard case of open systems with single-product industries. First of all, we easily see that the open model (2.1) can bc written in a form analogous to (3.1). We have:

$$
\left[\begin{array}{c}
\mathbf{x} \\
\hdashline L
\end{array}\right]=\left[\begin{array}{c:c}
\mathbf{T} & \frac{\mathbf{f}}{L} \\
\hdashline \mathbf{I} & 0
\end{array}\right]\left[\begin{array}{c}
\mathbf{x} \\
- \\
L
\end{array}\right]
$$

which, in obvious notation, can be rewritten as

$$
\mathbf{z}=\text { Mz. }^{2}
$$

For the corresponding price equation, we obtain: 


$$
\left[\begin{array}{l:l}
\mathbf{p} & 1
\end{array}\right]=\left[\begin{array}{l:l}
\mathbf{p} & 1
\end{array}\right]\left[\begin{array}{c:c}
\mathbf{T} & \frac{\mathbf{f}}{L} \\
\hdashline \mathbf{l} & 0
\end{array}\right]
$$

where we have standardized the wage $w$ at unit value. (We observe that the equation determining embodied labour times now is embedded in a larger system.) Consequently, again in obvious notation, we have for the dual system

$$
\mathbf{y}=\mathbf{y M}
$$

With $\mathbf{l}>0$ and $\mathbf{T}$ (semi)positive, we now have

Theorem 2. $\mathbf{M}$ is primitive.

Proof. We immediately have that there is no simultaneous permutation of rows and columns such that the sectors can be divided in successive clusters, each cluster delivering exclusively to the next one, the last cluster delivering exclusively to the first one. (For concepts used, see Takayama [6, pp. 376-378].) So, regardless of the character of T (primitive or imprimitive), $\mathbf{M}$ is primitive.

Now let us base a definition of embodiedness for this open system on the procedure of Section 3. That is, we consider $\mathbf{M}^{\infty} \equiv \lim _{t \rightarrow \infty} \mathbf{M}^{t}$ and identify its $(i$, $j)$ th element, $m_{i j}^{(\infty)}$, as the amount of commodity $i$ embodied in a unit of commodity $j$, etc. ${ }^{3}$ Now let us consider matrix $\mathbf{M}^{\infty}$ a bit closer. From Theorem 1, we have that the lefthand Frobenius eigenvector of $\mathbf{M}^{\infty}$ is equal to the corresponding eigenvector of $\mathbf{M}$. Recalling that $\mathbf{z}=[\mathbf{x} \mid L]^{\prime}$, matrix $\mathbf{M}^{\infty}$ then can be written (Theorem 1) as the outer product of the standardized vectors $\mathbf{y}$ and $\mathbf{z}$. We have $\mathbf{y z}=\left[\begin{array}{l|l|l}\mathbf{p} & 1\end{array}\right]\left[\begin{array}{lll}\mathbf{x} & \mathbf{L}\end{array}\right]^{\prime}=\mathbf{p x}+L \equiv \alpha$, say. So



$$
\begin{aligned}
& \equiv\left[\begin{array}{c:c}
\mathbf{M}_{11}^{(\infty)} & \mathbf{M}_{12}^{(\infty)} \\
\hdashline \mathbf{M}_{21}^{(\infty)} & \mathbf{M}_{22}^{(\infty)}
\end{array}\right] \text {. }
\end{aligned}
$$

We now have

$$
\begin{aligned}
& \text { Theorem } 3 . \mathbf{M}_{21}^{(\infty)}=(w L) \mathbf{l}^{*} . \\
& \text { Proof. } \mathbf{M}_{21}^{(\infty)}=(1 / \alpha) L \mathbf{p}=(1 / \alpha) L\left(\left.w\right|^{*}\right)=(1 / \alpha)(w L) \mathbf{l}^{*}
\end{aligned}
$$$$
\equiv \xi \mathbf{I}^{*} . \quad(\xi>0)
$$

So the vector of embodied labour times as calculated via our algorithm, that is the vector $\mathbf{M}_{21}^{(\infty)}$, is strictly proportional to the traditional vector of labour values I* of Section 2. Therefore, next to the usual "recipe" for calculating embodied labour (i.e. via the Leontief inverse), we now also have the above "Markovian" framework, which makes use of primitivity of the extended system matrix $\mathbf{M}$.

The last column of $\mathbf{M}^{\infty}$ may need some additional comments. Elements of this column represent the amounts of each commodity and labour "congealed" in the final demand basket $\mathbf{f}$. Because of the openness of the system, $f$ will vary following changes in the tastes and preferences of the human agents. Because total output $\mathbf{x}$ and the required labour force $L$ vary with $\mathbf{f}$, also the implied composite numeraire, being a scalar multiple of the commodity bundle $[\mathbf{x} \mid L]^{\prime}$, will change accordingly. ${ }^{4}$ (Compare here the situation for the closed model of Section 3, where the implied composite numeraire cannot vary.)

The difference with the usual imputation scheme, based on the Leontief inverse is quite clear. In the latter scheme, all inputs, labour included, are imputed to the production of the final demand vector. In our scheme, inputs are imputed to the production of each good, irrespcctive of its destination (intermediate or final use). Because of the proportionality of the vectors of embodied labour in both schemes (i.e. $\mathbf{M}_{21}^{(\infty)}$ in our scheme and $\mathrm{l}^{*}$ in the traditional scheme), prices in the open system thus can be interpreted in terms of both imputation schemes. We also have seen that (im)primitivity of the submatrix $\mathbf{T}$ (of $\mathbf{M}$ ) does not matter, matrix $\mathbf{M}$ itself being always primitive.

Our conclusion thus is that regardless of the composition of the final demand vector, also the open Leontief model can be rewritten to fit the framework of Section 3. Therefore, also here the production relations can be rewritten in a form analogous to (3.5).

\section{THE DYNAMIC CASE}

A most familiar specification of the dynamic Leontief model is the following form (see $[8,9,10]$ ):

$$
\mathbf{x}(\mathbf{t})=\mathbf{A x}(t)+\mathbf{B}[\mathbf{x}(t+1)-\mathbf{x}(t)]
$$

where $\mathbf{x}(t)$ stands for the gross output vector in period $t, \mathbf{A}$ for the matrix of intermediate inputs (including replacement investments), and $\mathbf{B}$ for the capital matrix. Solving for $\mathbf{x}(t+1)$, we obtain

$$
\mathbf{x}(t+1)=\left[\mathbf{I}+\mathbf{B}^{-1}(\mathbf{I}-\mathbf{A})\right] \mathbf{x}(t)
$$

The dual price equation, expressing equilibrium on each competitive market, is traditionally specified as:

$$
\mathbf{p}(t+1)[\mathbf{B}+\mathbf{I}-\mathbf{A}]=(1+r) \mathbf{p}(t) \mathbf{B}
$$

So

$$
\mathbf{p}(t+1)=(1+r) \mathbf{p}(t)\left[\mathbf{I}+(\mathbf{I}-\mathbf{A}) \mathbf{B}^{-1}\right]^{-1},
$$

where $\mathbf{p}(t)$ is the price vector during period $t$ and $r$ the rate of interest.

By now the above functional forms have been empirically implemented for many countries and many periods. One of the dominant characteristics of the above model is that it is plagued by the phenomenon of "dual (in)stability". That is, if the real system is relatively stable (i.e. if its output proportions converge to the balanced growth proportions), then the price system is relatively unstable. (As mentioned earlier, a vice-versa result also holds. See Solow [9], Jorgenson [10], Takayama [6], and Taylor [4] for the fundamentals of the discussion, Filippini [11] for a recent theoretical contribution, and Steenge [12] on orders of magnitude involved.)

However, if the economy grows along its von Neumann path of balanced growth, both the quantity and the price equation "behave," and reduce to

$$
\mathbf{x}(t)=(\mathbf{A}+\lambda \mathbf{B}) \mathbf{x}(t)
$$

and

$$
\mathbf{p}(t)=\mathbf{p}(t)[\mathbf{A}+\lambda \mathbf{B}],
$$


with $\lambda$ the rate of growth. (In this strict equilibrium case, we have $\lambda=r, 1 / \lambda$ being the Frobenius eigenvalue of matrix $(\mathbf{I}-\mathbf{A})^{-1} \mathbf{B}$. See further Takayama [6] and Leontief [8].) Writing $\mathbf{C} \equiv[\mathbf{A}+\lambda \mathbf{B}]$ and dropping the time index, we obtain the model of the previous sections:

$$
\mathbf{x}=\mathbf{C} \mathbf{x}
$$

and

$$
\mathbf{p}=\mathbf{p C}
$$

It will bc clear that in this case, pricc and output systems are consistent with each other in the sense discussed earlier. It will also be clear that if the system does not grow along its von Neumann ray, the dual (in)stability theorem applies, and prices or quantities may become negative. In that case, the economy does not satisfy the conditions for Saaty consistency.

Empirical work shows (Tokoyama and Murakami [13], Meyer and Schumann [14], Tsukui and Murakami [15], and many other sources) that the growth path of industrialized countries for extended periods can be described remarkably well by a simple balanced growth model. This suggests an interesting link with Stojanović's [16] matrix of growth. This (rank one) matrix can be written as:

$$
\mathbf{R}_{t}=\left[\begin{array}{cccc}
\Delta x_{1, t} & \Delta x_{1, t} & & \Delta x_{1, t} \\
---- & ---\cdots & \cdots & --- \\
x_{1, t} & x_{2, t} & & x_{n, t} \\
\cdots & \cdots & \cdots & \cdots \\
\cdots & \cdots & \cdots & \cdots \\
\Delta x_{n, t} & \Delta x_{n, t} & & \Delta x_{n, t} \\
--- & ---\cdots & --- \\
x_{1, t} & x_{2, t} & & x_{n, t}
\end{array}\right]
$$

with $\Delta x_{i, t}=x_{i, t}-x_{i, t-1}, i=1,2, \cdots n$. If the economy is in a state of balanced growth, we easily obtain (dropping the time index):

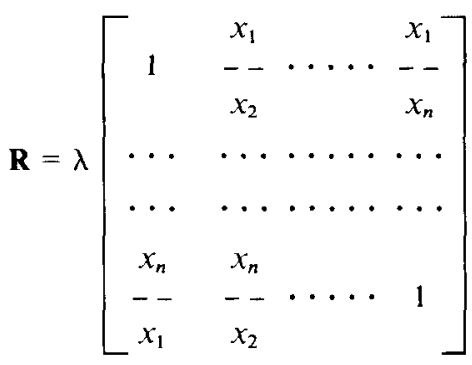

or

$$
\mathbf{R}=\lambda \mathbf{K},
$$

with $\lambda$ the rate of growth and $\mathbf{K}$ a reciprocal matrix of rank one. (See further Steenge [17] on these and some similar connections.)

The above shows that the balanced growth case can be described in terms of a priority matrix of rank one. This, however, suggests that violations of the property of reciprocity can be studied in terms of perturbations of this path, in terms of disruptions of the price system, or both. (Appropriate norms can be found in Saaty's publications; see also Lootsma [18] for some examples.) Note also that the usual scaling of input-output tables fits Saaty's numerical scale. For example, sectoral definitions normally are such that all output levels are of the same order of magnitude, their magnitudes not differing by more than a factor of 9 . Because of the easy conversion of the input-output framework to a Saaty framework, we may expect future research in this area to be fruitful.

\section{SUMMARY AND CONCLUSION}

Saaty's priority theory has given us a set of rules to assign weights (priorities) to a multiple of objects in a consistent way. The theory shows that eigenvalue-eigenvector procedures lead to "best" decisions in a certain well-defined sense. Saaty c.s. have convincingly shown that priority theory is useful in solving a number of economic problems. Here possibly the biggest advance is to be encountered in the field of multisector input-output analysis, where application of priority theory promises a substantial time- and labor-saving potential.

Traditional input-output theory imposes a strict equilibrium framework on the economy. Prices implied by the input-output framework are strict equilibrium prices. In fact, if we replace "weights" by "prices" and "objects" by "commodities" above, we have a description of what any price system is supposed to do. The Walrasian auctioneer, for example, is nothing but a device for consistently pricing goods. The Leontief systems consist of two separate systems, the quantity or primal system, and the price or dual system. In static analysis there are no problems of compatibility. Dynamic analysis, on the other hand, is invariably plagued by problems of compatibility of the two. Here an approach based on Saaty's priority analysis may be quite relevant, as this paper has set out to show. We were able to show that the various Leontief models can be reformulated in terms of frameworks involving eigenvalues and eigenvectors in an essential way. A matrix could be found, which was given an interpretation in terms of "composite numeraires" of specific proportions. This matrix could easily be linked to a Saaty priority matrix of rank one. This illustrates that an interpretation of the input-output relations according to the rules of priority theory points to the role of these particular numeraires as the binding element between price and quantity systems. Dynamic models were shown not to be consistent in this way, except in the special case of von Neumann growth. On the more general level, Saaty consistency analysis suggests that multisector theory has focused too much and too long on issues like decomposability vs. indecomposability: Properties like primitivity or imprimitivity may be just as essential for gaining insight in economic theories.

\section{NOTES}

1. For definitions, see Takayama [6, pp. 376-378]. One way of describing primitivity is that successive powers of matrix $g T$ (with $g$ a positive scalar such that $g T$ has Frobenius eigenvalue equal to unity) approach a certain positive limit matrix, while imprimitivity means that this is not the case; periodically the same matrix $g T$ will turn up. See also Theorem 2 for an interpretation in terms of the behavior of sector clusters. Note that Saaty's priority matrix is always primitive. 
2. To avoid an unnecessary proliferation of symbols, and because no confusion will arise with the exposition of Section 3, we have used the symbols $\mathbf{M}, \mathbf{z}$ and $\mathbf{y}$ also here to denote the main system matrix and the corresponding intensity and price vectors. Note again that $\{(4.2),(4.4)\}$ represents an open input-output system. Hence, the final demand vector $\mathbf{f}$ and the required labour force $L$ are variable.

3. Our procedure of arriving at embodied labour coefficients for this open system, being in line with Def. 1, therefore will also deviate from the standard approach of Section 2.

4. The fact that prices in the open Leontief system thus can be interpreted in terms of composite numeraires, does not detract from the fact that labour is the system's only primary factor; as mentioned, (changes in) tastes of the human agents in fact determine the (composition of) the composite numeraire. Because $\mathbf{x}$ varies with each $\mathbf{f}$, also the corresponding composite numeraire varies with each different $f$. This is in contrast to the case of the closed model of Section 3, where the consumption vector is fixed (and, consequently, also the corresponding composite numeraire).

\section{REFERENCES}

1. T. L. Saaty, The Analytic Hierarchy Process. McGrawHill, New York (1980).

2. T. I. Saaty and I. G. Vargas, Estimating technological coefficients by the analytic hierarchy process. Socio-Econ. Plan. Sci. 13, 333-336 (1979).

3. T. L. Saaty and J. M. Alexander, Thinking With Models. Pergamon Press, Oxford (1981).

4. L. Taylor, Theoretical foundations and technical implications. Ch. III in Ch. R. Blitzer, P. B. Clark and L. Taylor (eds): Economy-Wide Models and Development Planning. Oxford University Press, London (1975).

5. R. Dorfman, P. Samuelson and R. Solow (Dosso), Linear Programming and Economic Analysis. McGraw-Hill, New York (1958).

6. A. Takayama, Mathematical Economics. The Dryden Press, Hinsdale, IL (1974).

7. R. E. Miller and P. D. Blair, Input-Output Analysis: Foundations and Extensions. Prentice-Hall, Englewood Cliffs, NJ (1985).

8. W. W. Leontief, General numerical solution of the simple dynamic input-output system. Report on Research for 1953, Harvard Economic Research Project, 5-15, 160161. Harvard (1953).

9. R. M. Solow, Competitive valuation in a dynamic inputoutput system. Econometrica 27, 30-53 (1959).

10. D. Jorgenson, Stability of a dynamic input-output system. Review of Economic Studies 28, 105-116 (1961).

11. L. Filippini, Price and quantity adjustment in a dynamic closed model: The dual stability theorem. I. Macroeconomics 5, 185-196 (1983).

12. A. E. Steenge, On two types of stability in the dynamic Leontief model. Economics Letters 1, 105-109 (1978).

13. K. Tokoyama and Y. Murakami, Relative stability in two types of dynamic Leontief models. Int. Econ. Rev. 13, 408-415 (1972).

14. U. Meyer and J. Schumann, Das dynamische InputOutput Modell als Modell gleichgewichtgen Wachstums. Zeitschrift fur die gesamte Staatswissenschaft 133, 1-37 (1977).

15. J. Tsukui and Y. Murakami, Turnpike Optimality in Input-Output Systems. North-Holland, Amsterdam (1979).

16. D. Stojanović, The model based on the matrix of economic growth. Socio-Econ. Plan. Sci. 18, 167169 (1984).

17. A. E. Steenge, The verification of efficient growth: An approach via Stojanović's matrix of growth. J. Macroeconomics 3, 271-281 (1981).

18. F. A. Lootsma, Saaty's priority theory and the nomination of a senior professor in operations research. European Journal of Operations Research 4, 380-388 (1980).

19. Centraal Bureau voor de Statistiek, De produktie-structuur van de Nederlandse volkshuishouding. Deel V. Inputoutput tabellen 1965-1967 (in Dutch). Staatsuitgeverij, 's-Gravenhage (1971).

20. P. M. C. de Boer, Price effects in input-output relations: A theoretical and empirical study for The Netherlands, 1949-1967. Lecture Notes in Economics and Mathematical Systems, Vol 201. Spinger Verlag, Berlin Heidelberg (1982).

\section{APPENDIX}

In this appendix we shall illustrate the approach of this paper by means of an empirical example. We have employed the Dutch input-output table for the year 1967, compiled by the Dutch Central Bureau of Statistics (C.B.S.) [19]. The original C.B.S. table distinguishes 35 industrial sectors. However, for our purposes, an aggregated version of the table will be sufficient. Therefore, we have followed the aggregation scheme proposed by De Boer [20]. Here the Dutch economy is analyzed in terms of 11 productive sectors. Table 1, below, presents the aggregated input-output flow matrix. Column 12 lists the final deliveries, and column 13 total sectoral production. Correspondingly, row 12 gives us the required primary inputs. Straightforward calculation (Table 2) gives matrix $\mathbf{M}$, as it appears in system $\{(4.2),(4.4)\}$. (For example, $m_{12}=6307 /$ $20784=0.303$, etc.) Note that $\mathbf{M}$ has Frobenius eigenvalue equal to unity. Table 3 contains the elements of matrix $\mathbf{M}^{2}$. Columnwise, we already observe here a slight resemblance to the proportions of the total output vector. Matrix $\mathbf{M}^{\infty}$ for Holland is given in Table 4. (Because we are dealing with an actual input-output table in current prices, obviously, all columns of $\mathbf{M}^{\infty}$ are equal.) Finally, restandardization (Section 3) gives us matrix $\mathbf{K}$, Saaty's reciprocal matrix of rank one (Table 5). 
Table 1. Flow matrix, The Netherlands $1967^{* 1}$

\begin{tabular}{|c|c|c|c|c|c|c|c|c|c|c|c|c|c|}
\hline I & 955 & 6307 & 9 & 2 & 0 & 0 & 0 & 40 & 0 & 3 & 54 & 3414 & 10784 \\
\hline II & 2700 & 2781 & 9 & 133 & 2 & 0 & 0 & 18 & 1 & 24 & 662 & 14454 & 20784 \\
\hline II I & 23 & 17 & 1190 & 33 & 43 & 13 & 1 & 101 & 14 & 30 & 40 & 4738 & 6243 \\
\hline IV & 442 & 219 & 230 & 1249 & 315 & 412 & 161 & 343 & 148 & 289 & 298 & 7097 & 11203 \\
\hline V & 203 & 376 & 41 & 264 & 4887 & 1405 & 122 & 264 & 258 & 565 & 132 & 13821 & 22338 \\
\hline VI & 116 & 66 & 31 & 106 & 188 & 882 & 46 & 84 & 102 & 63 & 694 & 12439 & 14817 \\
\hline$V \perp I$ & 21 & 106 & 51 & 189 & 196 & 62 & 93 & 141 & 112 & 106 & 153 & 1696 & 2926 \\
\hline VIII & 98 & 488 & 188 & 558 & 458 & 1953 & 374 & 2184 & 572 & 120 & 381 & 3777 & 11151 \\
\hline IX & 291 & 674 & 23.5 & 298 & 644 & 717 & 40 & 478 & 243 & 122 & 152 & 13671 & 17565 \\
\hline $\mathrm{X}$ & 53 & 161 & 48 & 79 & 152 & 107 & 11 & 184 & 2595 & 553 & 409 & 6170 & 10522 \\
\hline$X I$ & 222 & 194 & 156 & 250 & 554 & 276 & 75 & 455 & 446 & 482 & 1298 & 13777 & 18185 \\
\hline$X I I$ & 5660 & 9395 & 4055 & 8042 & 14899 & 8990 & 2003 & 6859 & 13074 & 8165 & 13912 & 0 & 95054 \\
\hline
\end{tabular}

*) Sectoral classification: I Agriculture, forestry and fishing; II Food, beverages and tobacco; III Textiles, footwear and other wearing apparel; IV Chemicals, oil refineries; $V$ Metal industry; VI Construction; VII Public utilities;VIII other manufacturing industries; IX Trade (margins); $X$ Transport, storage and communication; XI Other service industries; XII Primary inputs.

Table 2. Matrix M, The Netherlands 1967

\begin{tabular}{|c|c|c|c|c|c|c|c|c|c|c|c|c|}
\hline I & 0.089 & 0.303 & 0.001 & 0.000 & 0.000 & 0.000 & 0.000 & 0.004 & 0.000 & 0.000 & 0.003 & 0.036 \\
\hline II & 0.250 & 0.134 & 0.001 & 0.012 & 0.000 & 0.000 & 0.000 & 0.002 & 0.000 & 0.002 & 0.036 & 0.152 \\
\hline I I I & 0.002 & 0.001 & 0.191 & 0.003 & 0.002 & 0.001 & 0.000 & 0.009 & 0.001 & 0.003 & 0.002 & 0.050 \\
\hline IV & 0.041 & 0.011 & 0.037 & 0.111 & 0.014 & 0.028 & 0.055 & 0.031 & 0.008 & 0.027 & 0.016 & 0.075 \\
\hline v & 0.019 & 0.018 & 0.007 & 0.024 & 0.219 & 0.095 & 0.042 & 0.024 & 0.015 & 0.054 & 0.007 & 0.145 \\
\hline VI & 0.011 & 0.003 & 0.005 & 0.009 & 0.008 & 0.060 & 0.016 & 0.008 & 0.006 & 0.006 & 0.038 & 0.131 \\
\hline VII & 0.002 & 0.005 & 0.008 & 0.017 & 0.009 & 0.004 & 0.032 & 0.013 & 0.006 & 0.010 & 0.008 & 0.018 \\
\hline VI 11 & 0.009 & 0.023 & 0.030 & 0.050 & 0.021 & 0.132 & 0.128 & 0.196 & 0.033 & 0.011 & 0.021 & 0.040 \\
\hline IX & 0.027 & 0.032 & 0.038 & 0.027 & 0.029 & 0.048 & 0.014 & 0.043 & 0.014 & 0.012 & 0.008 & 0.144 \\
\hline $\mathrm{x}$ & 0.005 & 0.008 & 0.008 & 0.007 & 0.007 & 0.007 & 0.004 & 0.017 & 0.148 & 0.053 & 0.022 & 0.065 \\
\hline$X I$ & 0.021 & 0.009 & 0.025 & 0.022 & 0.025 & 0.019 & 0.026 & 0.041 & 0.025 & 0.046 & 0.071 & 0.145 \\
\hline XI I & 0.525 & 0.452 & 0.650 & 0.718 & 0.667 & 0.607 & 0.685 & 0.615 & 0.744 & 0.776 & 0.765 & 0.000 \\
\hline
\end{tabular}

Table 3. Matrix $\mathbf{M}^{2}$, The Netherlands 1967

\begin{tabular}{|c|c|c|c|c|c|c|c|c|c|c|c|c|}
\hline I & 0.103 & 0.084 & 0.024 & 0.030 & 0.024 & 0.022 & 0.025 & 0.024 & 0.027 & 0.029 & 0.039 & 0.050 \\
\hline II & 0.137 & 0.163 & 0.101 & 0.113 & 0.103 & 0.094 & 0.106 & 0.097 & 0.115 & 0.121 & 0.124 & 0.036 \\
\hline I I I & 0.027 & 0.024 & 0.069 & 0.037 & 0.034 & 0.032 & 0.036 & 0.034 & 0.038 & 0.040 & 0.039 & 0.011 \\
\hline IV & 0.052 & 0.051 & 0.062 & 0.070 & 0.057 & 0.057 & 0.065 & 0.060 & 0.063 & 0.065 & 0.064 & 0.026 \\
\hline v & 0.090 & 0.080 & 0.101 & 0.116 & 0.148 & 0.118 & 0.116 & 0.103 & 0.122 & 0.130 & 0.120 & 0.058 \\
\hline VI & 0.073 & 0.064 & 0.088 & 0.097 & 0.091 & 0.086 & 0.094 & 0.085 & 0.100 & 0.105 & 0.106 & 0.018 \\
\hline VII & 0.012 & 0.011 & 0.015 & 0.017 & 0.015 & 0.015 & 0.017 & 0.015 & 0.016 & 0.016 & 0.016 & 0.008 \\
\hline VIII & 0.035 & 0.032 & 0.043 & 0.050 & 0.040 & 0.064 & 0.063 & 0.070 & 0.041 & 0.040 & 0.045 & 0.048 \\
\hline IX & 0.089 & 0.080 & 0.104 & 0.111 & 0.105 & 0.100 & 0.108 & 0.101 & 0.112 & 0.116 & 0.116 & 0.026 \\
\hline $\mathrm{X}$ & 0.042 & 0.038 & 0.051 & 0.053 & 0.051 & 0.051 & 0.050 & 0.052 & 0.059 & 0.057 & 0.055 & 0.033 \\
\hline$X I$ & 0.085 & 0.077 & 0.105 & 0.113 & 0.106 & 0.100 & 0.110 & 0.104 & 0.119 & 0.121 & 0.120 & 0.030 \\
\hline XII & 0.256 & 0.297 & 0.236 & 0.193 & 0.227 & 0.260 & 0.210 & 0.256 & 0.189 & 0.161 & 0.156 & 0.655 \\
\hline
\end{tabular}


Table 4. Matrix $\mathbf{M}^{\infty}$, The Netherlands 1967

\begin{tabular}{|c|c|c|c|c|c|c|c|c|c|c|c|c|}
\hline 1 & 0.045 & 0.045 & 0.045 & 0.045 & 0.045 & 0.045 & 0.045 & 0.045 & 0.045 & 0.045 & 0.045 & 0.045 \\
\hline I I & 0.086 & 0.086 & 0.086 & 0.086 & 0.086 & 0.086 & 0.086 & 0.086 & 0.086 & 0.086 & 0.086 & 0.086 \\
\hline I I I & 0.026 & 0.026 & 0.026 & 0.026 & 0.026 & 0.026 & 0.026 & 0.026 & 0.026 & 0.026 & 0.026 & 0.026 \\
\hline IV & 0.046 & $0.01,6$ & 0.046 & 0.046 & 0.046 & 0.046 & 0.046 & 0.046 & 0.046 & 0.046 & 0.046 & 0.046 \\
\hline v & 0.092 & 0.092 & 0.092 & 0.092 & 0.092 & 0.092 & 0.092 & 0.092 & 0.092 & 0.092 & 0.092 & 0.092 \\
\hline VI & 0.061 & 0.061 & 0.061 & 0.061 & 0.061 & 0.061 & 0.061 & 0.061 & 0.061 & 0.061 & 0.061 & 0.061 \\
\hline VII & 0.012 & 0.012 & 0.012 & 0.012 & 0.012 & 0.012 & 0.012 & 0.012 & 0.012 & 0.012 & 0.012 & 0.012 \\
\hline VIII & 0.046 & 0.046 & 0.046 & 0.046 & 0.046 & 0.046 & 0.046 & 0.046 & 0.046 & 0.046 & 0.046 & 0.046 \\
\hline IX & 0.073 & 0.073 & 0.073 & 0.073 & 0.073 & 0.073 & 0.073 & 0.073 & 0.073 & 0.073 & 0.073 & 0.073 \\
\hline $\mathrm{x}$ & 0.044 & 0.044 & 0.044 & 0.044 & 0.044 & 0.044 & 0.044 & 0.044 & 0.044 & 0.044 & 0.044 & 0.044 \\
\hline XI & 0.075 & 0.075 & 0.075 & 0.075 & 0.075 & 0.075 & 0.075 & 0.075 & 0.075 & 0.075 & 0.075 & 0.075 \\
\hline$X I I$ & 0.393 & 0.393 & 0.393 & 0.393 & 0.393 & 0.393 & 0.393 & 0.393 & 0.393 & 0.393 & 0.393 & 0.393 \\
\hline
\end{tabular}

Tabie 5. Matrix K, The Netherlands 1967

\begin{tabular}{|c|c|c|c|c|c|c|c|c|c|c|c|c|}
\hline I & 1 & 0.519 & 1.727 & 0.963 & 0.483 & 0.728 & 3.686 & 0.967 & 0.614 & 1.025 & 0.593 & 0.113 \\
\hline II & 1.927 & 1 & 3.329 & 1.855 & 0.930 & 1.403 & 7.103 & 1.864 & 1.183 & 1.975 & 1.143 & 0.219 \\
\hline I II & 0.579 & 0.300 & 1 & 0.557 & 0.279 & 0.421 & 2.134 & 0.560 & 0.355 & 0.593 & 0.343 & 0.066 \\
\hline IV & 1.039 & 0.539 & 1.794 & 1 & 0.502 & 0.756 & 3.839 & 1.005 & 0.638 & 1.065 & 0.616 & 0.118 \\
\hline v & 2.071 & 1.075 & 3.578 & 1.994 & 1 & 1.508 & 7.634 & 2.003 & 1.272 & 2.123 & 1.228 & 0.235 \\
\hline V 1 & 1.374 & 0.713 & 2.373 & 1.323 & 0.663 & 1 & 5.064 & 1.329 & 0.844 & 1.408 & 0.815 & 0.156 \\
\hline VII & 0.271 & 0.141 & 0.469 & 0.261 & 0.131 & 0.197 & 1 & 0.262 & 0.167 & 0.278 & 0.161 & 0.031 \\
\hline VIII & 1.034 & 0.537 & 1.786 & 0.995 & 0.499 & 0.753 & 3.811 & 1 & 0.635 & 1.060 & 0.613 & 0.117 \\
\hline IX & 1.629 & 0.845 & 2.814 & 1.568 & 0.786 & 1.185 & 6.003 & 1.575 & 1 & 1.699 & 0.966 & 0.185 \\
\hline $\mathrm{x}$ & 0.976 & 0.506 & 1.685 & 0.939 & 0.471 & 0.710 & 3.596 & 0.944 & 0.599 & 1 & 0.579 & 0.111 \\
\hline$x I$ & 1.686 & 0.875 & 2.913 & 1.623 & 0.814 & 1.227 & 6.215 & 1.631 & 1.035 & 1.728 & 1 & 0.191 \\
\hline$X I I$ & 8.814 & 4.573 & 1.226 & 8.485 & 4.255 & 6.415 & 32.486 & 8.524 & 5.412 & 9.034 & 5.227 & 1 \\
\hline
\end{tabular}

